\title{
Dynamics of $\mathrm{He}$ atoms adsorbed on a carbon nanotube
}

\author{
Mikhail A. Strzhemechny and Irina V. Legchenkova \\ B. Verkin Institute for Low Temperature Physics and Engineering of the National Academy of Sciences of Ukraine \\ 47 Lenin Ave., Kharkov 61103, Ukraine \\ E-mail: strzhemechny@ilt.kharkov.ua \\ Received February 22, 2011
}

\begin{abstract}
The spectrum of a helium atom adsorbed on an armchair single-walled carbon nanotube is calculated. It is shown that a helium adatom tunnels over the surface of the nanotube within a band $10 \mathrm{~K}$ (for ${ }^{4} \mathrm{He}$ ) to $14 \mathrm{~K}$ (for ${ }^{3} \mathrm{He}$ ) wide. The undersaturated system of helium adatoms on nanotubes can be used as another system for studying quantum transport. It is also felt that this dynamics can be an important factor for the explanation of the large negative expansivities of nanotube bundles with adsorbed helium.
\end{abstract}

PACS: 81.05.ub Nanotubes;

68.43.-h Chemisorption/physisorption: adsorbates on surfaces;

65.40.-b Thermal properties of crystalline solids.

Keywords: nanotubes, helium atoms, energy spectrum.

Adsorption properties of bundles of carbon nanotubes, especially with lighter species like hydrogen and helium isotopes as adsorbates, attract interest due to the possibility [1] of creating low-dimensional condensed systems with unusual quantum behavior [2-4]. Low-temperature dilatometry of single-walled carbon nanotubes (SWNT) saturated with ${ }^{4} \mathrm{He}$ showed [5] a considerable (as compared to pure nanotubes) increase of the negative thermal expansivity below $4 \mathrm{~K}$. Despite the lack of a specific physical mechanism responsible for this increase, the authors tentatively ascribed this effect to the tunneling nature of the helium subsystem. The recent similar experiments of the same group on SWNT samples with adsorbed ${ }^{3} \mathrm{He}$ revealed [6] a further huge increase of the negative expansivity at low temperatures. When considered together, these two results add weight to the assumption of the crucial role of tunneling due to the quantum nature of $\mathrm{He}$ atoms. It should be noted that the total content of helium in both cases mentioned above amounted to a helium-to-carbon atomic ratio below 0.1 , which makes the $\mathrm{He}-\mathrm{He}$ interaction a less important factor and allows description of the helium subsystem as a set of independent particles in the potential relief of a nanotube. The aim of this communication is to consider the quantum dynamics of helium atoms adsorbed on the nanotube surface in an attempt to understand the abovementioned low-temperature phenomena.

From scratch we preferred to deal with a certain specific model of SWNT, which would provide a good general understanding of the problem in question. To start with, we had at our disposal the potential curves [7] of a $\mathrm{He}$ atom interacting with the outer and inner surfaces of a $(12,12)$ armchair SWNT. We restrict our consideration to the outer surface, the respective potential curves being shown in Fig. 1.

We are to deal with a quantum-mechanical eigenvalue problem of cylindric symmetry described by the Schrödinger equation

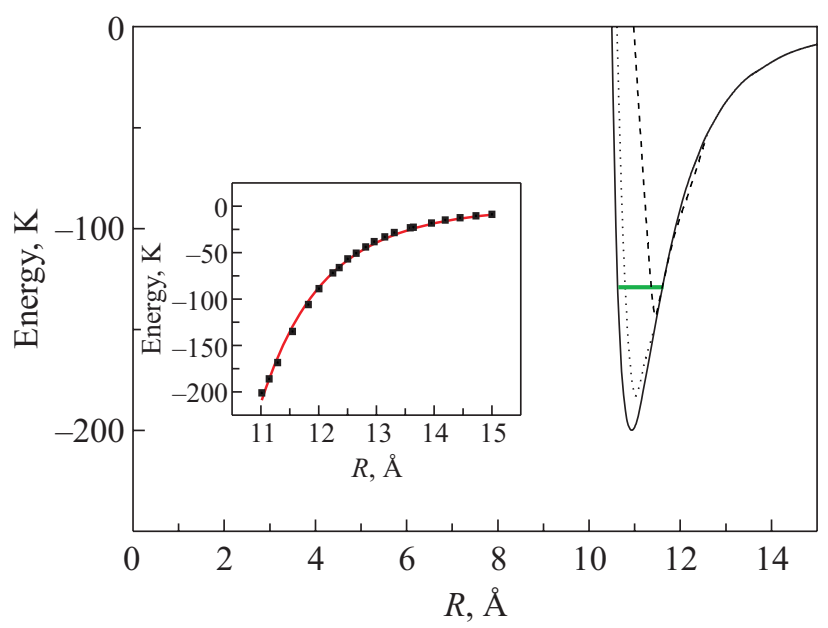

Fig. 1. Interaction energy of an outside $\mathrm{He}$ atom with a nanotube of $16.27 \AA$ in diameter [7]. The distance is counted from the nanotube center normal to the tube axis and going through: a hexagon center (solid), carbon atom (dashed), and center of $\mathrm{C}-\mathrm{C}$ bond (dotted). The thick horizontal line indicates the radial ground state energy level. The inset: analytical approximation of the first among the three potential curves. 


$$
-\frac{\hbar^{2}}{2 m}\left[\frac{1}{R} \frac{\partial}{\partial R}\left(R \frac{\partial}{\partial R}\right)+\frac{1}{R^{2}} \frac{\partial^{2}}{\partial \phi^{2}}+\frac{\partial^{2}}{\partial z^{2}}\right] \Psi+U(R, z, \phi) \Psi=E \Psi .
$$

Here $R$ is the distance from the outer nanotube surface, $z$ is the coordinate along the tube axis, and $\phi$ is the circumferential angle coordinate. The actual potential energy $U(R, z, \phi)$ does not separate into a sum of terms each of which depends only on one variable. However, there are reasonable arguments which allow us to approximate $U(R, z, \phi)$ as

$$
U(R, z, \phi)=V(R)+u(z, \phi),
$$

where $u(z, \phi)$ is a periodic function in two dimensions, which will be treated in more detail below. Indeed, since all slopes of the three potential curves in Fig. 1 are steep, the uniformly continued function $u(z, \phi)$ will add almost nothing to the wave function outside the potential well and will distort but slightly the real shapes of both the true $U(R, z, \phi)$ and the wave function. But as soon as the potential surface is represented as in Eq. (2), the variables separate and Eq. (1) can be easily solved. In order to do that analytically, we have to approximate both functions in Eq. (2). We took $V(R)$ in the form

$$
V(R)= \begin{cases}\infty, & R=R_{0} \simeq 11 \AA, \\ A / R^{N}, & R>R_{0},\end{cases}
$$

where the fitting parameters are $N \simeq 10 ; A \simeq-7 \cdot 10^{12} \mathrm{~K} \cdot \AA^{10}$ (cf. the fit in the inset in Fig. 1). We used the variational approach to evaluate the energy of the ground state for the potential of (3) to find that the most appropriate trial function has the form $\left(R-R_{0}\right) \exp \left(-\alpha\left(R-R_{0}\right)^{2}\right)$ with $\alpha \simeq 2.0404 \AA^{-2}$, which means that the wave function, shown in Fig. 2 is centered at $\bar{R} \simeq 11.52 \AA$ with a characteristic width of about $0.70 \AA$. The relevant energy level of $-131.0 \mathrm{~K}$ is shown in Fig. 1 as a thick horizontal line. Given this rather sharp shape of the radial wave function, the $1 / R^{2}$ factor in front of the $\partial^{2} / \partial \phi^{2}$ operator can be substituted by a constant, which will bring about an insignificant shift to the ground state energy upon account of the remaining periodic part of the Hamiltonian.

For the specific nanotube geometry considered here the periodic potential energy can be represented in the form

$$
u(z, \phi)=u_{0}\left[\cos \frac{2 \pi z}{L}+\cos \left(\frac{\pi z}{L}+\frac{\sqrt{3} \pi \bar{R} \phi}{L}\right)\right] .
$$

Here $u_{0} \simeq 7.5 \mathrm{~K}, L=2.05 \AA$ is the natural period, namely, the distance between the centers of opposite $\mathrm{C}-\mathrm{C}$ bonds in a hexagon, and $\bar{R} \simeq 11.52 \AA$, which is slightly larger than $R_{0}$ and thus this difference can be disregarded. It should be also noted that $2 \pi \bar{R} \simeq N L \sqrt{3} / 2$ where $N=12$ is the number of hexagon strips along our specific armchair nanotube.

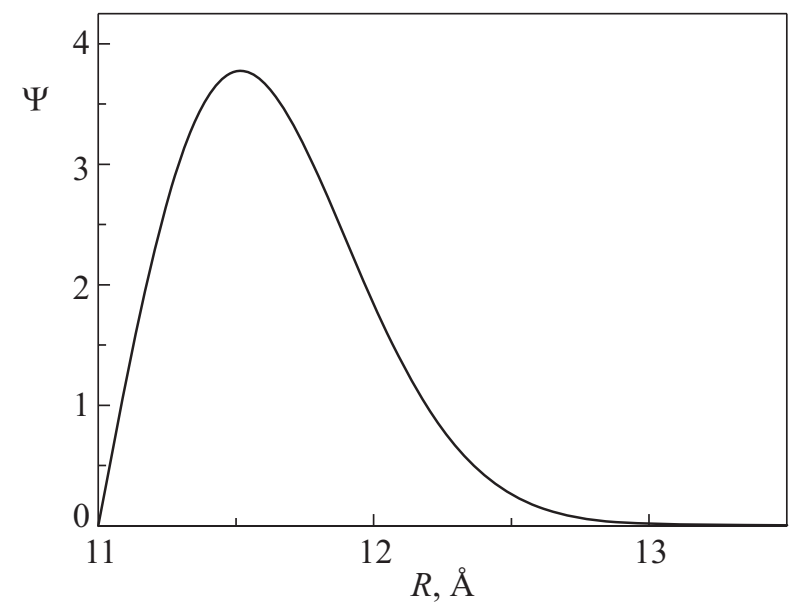

Fig. 2. The normalized partial wave function $\Psi(R)$.

In order to get insight into the problem of the variables $z$ and $\phi$, we solve an auxiliary problem. Ignoring for the time being the circumferential degree of freedom, we approximate the periodic potential along the line that unites neighbor carbon hexagon centers and goes through the center of the $\mathrm{C}-\mathrm{C}$ bond common for these two neighbor hexagons:

$$
u(z)=u_{0} \cos \frac{2 \pi z}{L}
$$

Now we transform Eq. (1) to dimensionless variables, making sure that the Schrödinger equation for the periodic function $u(z)$ is in the traditional dimensionless form of the Mathieu equation [8]

$$
\frac{d^{2} u(\zeta)}{d \zeta^{2}}+(p-2 q \cos 2 \zeta) u(\zeta)=0 .
$$

Here $\zeta=\xi / a_{0}$ with $a_{0}=L / \pi \simeq 0.652 \AA ; p=E / \varepsilon_{0}$ is the dimensionless energy eigenvalue with

$$
\varepsilon_{0}=\frac{\hbar^{2}}{2 m a_{0}^{2}} \simeq 14.26 \mathrm{~K}
$$

and $q=u_{0} / 2 \varepsilon_{0} \simeq 0.263$ is the dimensionless amplitude of the spatial variation of the potential relief. The lowest eigenstate of Eq. (6) is described by the Mathieu function $c e_{0}(\zeta, q)$. For sufficiently small $q$ this periodic function can be represented [8] as an expansion in powers of $q$

$$
c e_{0}(\zeta, q)=\frac{1}{\sqrt{2}}\left[1-\frac{q}{2} \cos 2 \zeta+q^{2}\left(\frac{\cos 4 \zeta}{32}-\frac{1}{16}\right)-\ldots\right] \text {. }
$$

The third term can be omitted since for the actual values of the parameters involved the ratio of the third term to a sum of the first two varies in a periodic fashion between -0.0065 and -0.0019 . The respective eigenvalue $a_{0}(q)$, which is the energy shift, can also be estimated from the expression [8] $a_{0}(q)=-q^{2} / 2+7 q^{2} / 128-\ldots \simeq-0.0343$, which to a good accuracy amounts to $-0.49 \mathrm{~K}$. 
The simple cosine form of the wave function in Eq. (6) is indicative of the fact that the actual approximation for the lowest band is a tight-binding one. This conclusion simplifies the task of calculating the dispersion law of the ${ }^{4} \mathrm{He}$ atom tunneling over the periodically corrugated cylindrical surface of a SWNT. Then the effective tunneling Hamiltonian $H_{t}$ can be cast in the form

$$
H_{t}=\frac{J}{2} \sum_{<i, j>} a_{i}^{+} a_{j},
$$

where summation runs over nearest neighbors and the jump frequency $J$ in absolute values is $5 \mathrm{~K}$ for ${ }^{4} \mathrm{He}$ and $7 \mathrm{~K}$ for ${ }^{3} \mathrm{He}$, respectively. The spectrum $\varepsilon_{n}(k)$, which is easily calculable, is quasi-continuum along $z$ and quantized for lateral motion:

$$
\varepsilon_{n}(k)=J[\cos 2 \pi k L+2 \cos \pi k L \cos \pi n / N] .
$$

For the specific type of SWNT, $N=6$ and $1 \geq n \geq 6$, and the spectrum consists of three pairs of degenerate bands. The width of the main band is $10.1 \mathrm{~K}$ for ${ }^{4} \mathrm{He}$ atoms and $14.3 \mathrm{~K}$ for ${ }^{4} \mathrm{He}$ atoms.

In conclusion, we point out that the experiments which initiated this short note clearly suggest that a system of undersaturated helium atoms on carbon nanotubes can be viewed as a new quantum tunneling system enabling studies of quantum transport of solitary adatoms.

The authors thank V.G. Manzhelii and A.V. Dolbin for valuable discussions and K.A. Chishko for critical reading of the manuscript.

1. M.M. Calbi, F. Toigo, and W.W. Cole, Phys. Rev. Lett. 86, 5062 (2001).

2. S.O. Diallo, B. Fåk, M.A. Adams, O.E. Vilches, M.R. Johnson, H. Schober, and H.R. Glyde, EPL 88, 56005 (2009).

3. T.N. Antsygina, I.I. Poltavsky, K.A. Chishko, T.A. Wilson, O.E. Vilches, and O.E. Vilches, Fiz. Nizk. Temp. 31, 1328 (2006) [Low Temp. Phys. 31, 1007 (2006)].

4. T.N. Antsygina, I.I. Poltavsky, and K.A. Chishko, J. Low Temp. Phys. 138, 223 (2005).

5. A.V. Dolbin, V.B. Esel'son, V.G. Gavrilko, V.G. Manzhelii, N.A. Vinnikov, S.N. Popov, and B. Sundqvist, Fiz. Nizk. Temp. 36, 797 (2010) [Low Temp. Phys. 36, 635 (2010)].

6. A.V. Dolbin, V.B. Esel'son, V.G. Gavrilko, V.G. Manzhelii, N.A. Vinnikov, S.N. Popov, and B. Sundqvist, Fiz. Nizk. Temp. 37, 685 (2011) [Low Temp. Phys. 37, No. 6 (2011)].

7. L. Firlej and B. Kuchta, Colloids and Surfaces A: Physicochem. Eng. Aspects 241, 149 (2004).

8. G. Blanch, Mathieu Functions in: Handbook of Mathematical Functions, M. Abramowitz and I.E. Stegun (eds.), NBS Appl. Math. Ser. 55 (1964). 